\title{
Spatial Graphs and Oriented Matroids: the Trefoil ${ }^{*}$
}

\author{
J. L. Ramírez Alfonsín \\ Instituto de Matemáticas, Universidad Nacional Autónoma de México, \\ Area de la Investigación Cientifica, Circuito Exterior C.U., Mexico D.F. 04510
}

\begin{abstract}
Let $m=m(K)$ be the smallest positive integer such that every linear spatial representation of the complete graph with $n$ vertices, $n \geq m$, contains either the knot $K$ or its mirror. In this paper we show that $m$ (Trefoil $)=7$. The proof uses the theory of oriented matroids.
\end{abstract}

\section{Introduction}

A link $L$ with $k$ components consists of $k$ disjoint simple smooth closed curves in $R^{3}$. A knot $K$ is a link with one component. We say that two links $K$ and $L$ are isotopic if there exists a homotopy $h_{t}: S^{3} \longrightarrow S^{3}(0 \leq t \leq 1)$ such that $h_{0}=1$, each $h_{i}$ is a homeomorphism, and $h_{1}(K)=L$. We restrict our attention to tame knots. A link diagram $D(K)$ is obtained from $L$ by projecting it into a plane in such a way that the projection of each component is smooth and at most two curves intersect at any point. At each crossing point of the link diagram the curve which goes over the other is specified, see Fig. 2.

The fundamental theorem of Reidemeister [10] states:

Theorem 1.1. Two links $K$ and $L$ are isotopic if and only if any link diagram $D(K)$ can be transformed into any link diagram $D(L)$ by a finite sequence of the moves I, II, III and their inverses (see Fig. 1).

The mirror of a knot $K$, denoted by $K^{*}$, is obtained by a reflection of $K$ in a plane.

\footnotetext{
* This work was done while the author was doing a Postdoc visiting the Université Pierre et Marie Curie, Paris 6, Equipe Combinatoire. His current mail address is Université Pierre et Marie Curie, Paris 6, Equipe Combinatoire - Case 189, 4 Place Jussieu, Paris 75252 Cedex 05, France.
} 
I

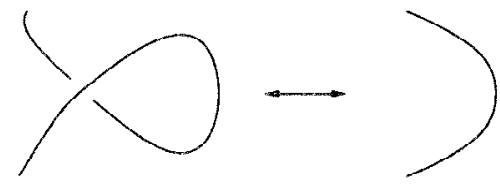

II
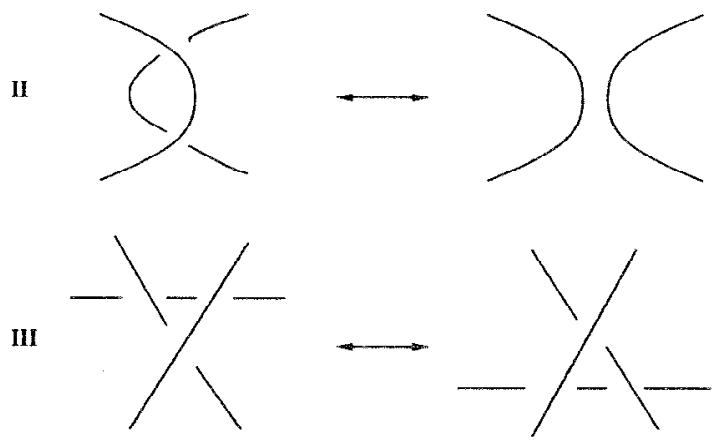

Fig. 1. Reidemeister moves.

A knot $K$ is called amphicheiral if $K$ is isotopic to $K^{*}$ (for further definitions and terminology on knot theory refer to [3]).

Note that the Trefoil, $T$ (Fig. 2(a)), and its mirror, $T^{*}$ (Fig. 2(b)), are not isotopic; there is no sequence of Reidemeister moves which will transform $T$ to $T^{*}$, that is, the Trefoil is nonamphicheiral, see [5]. It is known [10] that six straight lines are necessary and sufficient to contruct $T$ in $R^{3}$.

A graph is complete if every pair of vertices is adjacent. A complete graph with $n$ vertices is denoted by $\mathbf{K}_{n}$. A graph is a cycle if the union of all edges forms a closed path (see [2] for further graph theory details).

A spatial representation $\mathcal{R}(G)$, of a graph $G$, is the embedded image of $G$ in $R^{3}$, that is, the vertices of $G$ are distinct points in $R^{3}$ and the edges are simple Jordan curves

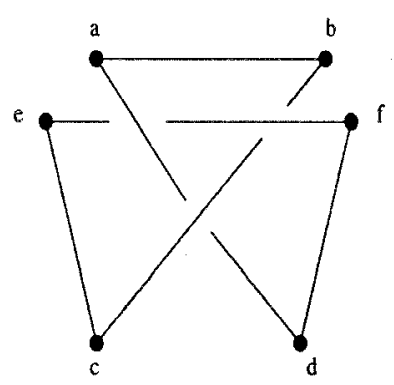

(a)

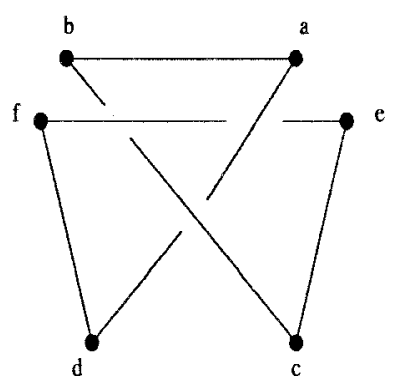

(b)

Fig. 2. The trefoil and its mirror. 
between them in such a way that any two curves are either disjoint or meet at a common point. We understand $\mathcal{R}(G)$ as a polygonal representation of $G$, see [3]. $\mathcal{R}(G)$ is linear if each edge is a straight line segment. Spatial cycles are often regarded as simple closed curves in $R^{3}$. Hence, we may regard spatial cycles as a link in $R^{3}$.

Sacks [14] proved that any linear $\mathcal{R}\left(\mathbf{K}_{6}\right)$ contains cycles isotopic to $2_{1}^{2}$ (the simplest nontrivial link). In a series of three papers, Robertson et al. [11]-[13] strengthened the latter by proving Sacks' conjecture [14] that a graph can be embedded in threedimensional space so that it contains no nontrivial link if and only if it contains as a minor none of the seven graphs (called the Petersen family in [12]) obtainable from $\mathbf{K}_{6}$ by $Y-\Delta$ and $\Delta-Y$ exchanges.

Here, we intend to continue similar investigations. Let $m(K)$ be the smallest positive integer such that every linear $\mathcal{R}\left(\mathbf{K}_{n}\right), n \geq m$, contains either the knot $K$ or $K^{*}$. Note that Sacks' result can be stated as $m\left(2_{1}^{2}\right)=6$ since $2_{1}^{2}$ is isotopic to its mirror. Our main result in this paper is the following.

Theorem 1.2. $m($ Trefoil $)=7$.

An old result by Conway and Gordon [4] states that any $\mathcal{R}\left(\mathbf{K}_{7}\right)$ (not necessarily linear) contains a nontrivial knot. More precisely, they proved that any $\mathcal{R}\left(\mathbf{K}_{7}\right)$ contains a Hamiltonian cycle with nonzero Arf invariant. Notice that their method does not ensure the existence of either the Trefoil or its mirror if $\mathcal{R}\left(\mathbf{K}_{7}\right)$ is linear. Therefore, Theorem 1.2 implies this result for linear embeddings.

Negami [9] showed that for any given knot, link, or $\mathcal{R}(G)$, (not necessarily linear) of a graph $G$, there is a sufficiently large complete graph $\mathbf{K}_{n}$ such that every linear $\mathcal{R}\left(\mathbf{K}_{n}\right)$ always contains a subdivision of $\mathcal{R}(G)$. In [8] Miyauchi generalizes this result for complete multipartite graphs and conjectures that any $\mathcal{R}\left(\mathbf{K}_{8}\right)$ (not necessarily linear) contains $T$.

Our technique to prove Theorem 1.2 lies completely on the theory of oriented matroids. The theory of oriented matroids provides a broad setting to describe geometric configurations. This theory considers the structure of dependencies in vector spaces over ordered fields.

Indeed, it is well known that there is a natural way to associate an oriented matroid (a set of circuits) to a given configuration of points. Thus, in order to prove Theorem 1.2, we may proceed as follows. Let $\mathcal{M}\left[\mathcal{R}\left(\mathbf{K}_{n}\right)\right]$ be the oriented matroid associated to the set of points of a linear $\mathcal{R}\left(\mathbf{K}_{n}\right)$. We propose three sets of conditions (in terms of circuits) and show that if $\mathcal{M}\left[\mathcal{R}\left(\mathbf{K}_{7}\right)\right]$ satisfies at least one of these conditions, then $\mathcal{R}\left(\mathbf{K}_{7}\right)$ is forced to contain a cycle isotopic to either $T$ or $T^{*}$.

Finally, with help of a computer program, we are able to check that $\mathcal{M}\left[\mathcal{R}\left(\mathbf{K}_{7}\right)\right]$ satisfies at least one of the above conditions for any linear $\mathcal{R}\left(\mathbf{K}_{7}\right)$ (possibly after a suitable permutation of elements). Also, we show that the analogue for $\mathbf{K}_{6}$ is not true. Hence, $m($ Trefoil $)=7$. We mention as well that we found that there exist linear $\mathcal{R}\left(\mathbf{K}_{7}\right)$ not containing either $T$ or $T^{*}$ using only six of the vertices.

The paper is organized as follows: In the next section we introduce briefly some basic notions of oriented matroids needed for the rest of the paper. In Section 3 we give the proof of Theorem 1.2. Finally, in the Appendix we discuss the checking conditions program. 


\section{Oriented Matroid Session}

We give some basic definitions of oriented matroids (see [1]). A signed set is a set $X$ together with a partition into two distinguished subsets $X^{+}$and $X^{-}$. The opposite of $X$ is the signed set $-X$ such that $(-X)^{+}=X^{-}$and $(-X)^{-}=X^{+}$.

An oriented matroid $\mathcal{M}$ on a finite set $E$ is defined by its collection $\mathcal{C}$ of signed circuits satisfying the following two properties:

1. For all $C_{1} \in \mathcal{C}, C_{1} \neq \emptyset$, and $-C_{1} \in \mathcal{C}$, and for all $C_{1}, C_{2} \in \mathcal{C}, C_{2} \subseteq C_{1}$ implies $C_{2}=C_{1}$ or $-C_{1}$

2. (Elimination property) For all $C_{1}, C_{2} \in \mathcal{C}$ with $C_{1} \neq-C_{2}$ and all $x \in\left(C_{1}^{+} \cap C_{2}^{-}\right)$, there exists $C_{3} \in \mathcal{C}$ such that $C_{3}^{+} \subseteq\left(C_{1}^{+} \cup C_{2}^{+}\right) \backslash x$ and $C_{3}^{-} \subseteq\left(C_{1}^{-} \cup C_{2}^{-}\right) \backslash x$.

By ignoring signs, a (nonoriented) underlying matroid $\underline{\mathcal{M}}$ is clearly attached to each oriented matroid $\mathcal{M}$. The bases of $\mathcal{M}$ are the maximal subsets of $E$ which contain no circuit, that is, they are the bases of $\underline{\mathcal{M}}$. The rank function of $\mathcal{M}$ is the rank function of $\mathcal{M}$ and is denoted by $r$.

Let $A$ be a subset of $E$. We set ${ }_{-A} \mathcal{C}=\left\{{ }_{-A} C: C \in \mathcal{C}\right\}$, where ${ }_{-A} C$ is the signed set with $\left({ }_{-A} C\right)^{+}=\left(C^{+} \backslash A\right) \cup\left(C^{-} \cap A\right)$ and $\left({ }_{-A} C\right)^{-}=\left(C^{-} \backslash A\right) \cup\left(C^{+} \cap A\right)$. It is clear from the axioms that ${ }_{-A} \mathcal{C}$ is also a set of circuits of an oriented matroid, denoted by ${ }_{-A} \mathcal{M}$. We say that ${ }_{-A} \mathcal{M}$ is obtained from $\mathcal{M}$ by reorientation on $A$. The equivalence classes for the relation $\mathcal{M} \sim \mathcal{M}^{\prime}$ if and only if there exists $A \subseteq E$ such that $\mathcal{M}^{\prime}={ }_{-A} \mathcal{M}$ are called orientation classes.

Another characterization of oriented matroids was given by Lawrence [7] in terms of basis orientations. More formally, let $r$ be an integer $\geq 1$ and let $E=\{1, \ldots, n\}$ be a set. A mapping $\chi: E^{r} \longrightarrow\{-1,0,+1\}$ is a basis orientation of an oriented matroid of rank $r$ on $E$ if and only if $\chi$ is a chirotope, that is, a special alternating mapping not identically zero (see also [6] for a different characterization of oriented matroids). An oriented matroid $\mathcal{M}$ is uniform if $\chi: E^{r} \longrightarrow\{-1,+1\}$. Finally, we use the following notation: $\left(e_{1}, \bar{e}_{2}, \bar{e}_{3}, e_{4}, \bar{e}_{5}\right)$ denotes the signed set $X$ with $X^{+}=\left\{e_{1}, e_{4}\right\}$ and $X^{-}=\left\{e_{2}, e_{3}, e_{5}\right\}$.

A fundamental example of oriented matroids [1] is the following. Let $V$ denote a finite set of points spanning $R^{d}$ and let $\mathcal{C}$ be the collection of signed subsets $C$ of $V$ which are inclusion-minimal with the property $C \neq \emptyset$ and there is an affine combination $\sum_{x \in C} \alpha(x) \cdot x=0, \sum_{x \in C} \alpha(x)=0$ such that

$$
C^{+}=\{x \in C, \alpha(x)>0\} \quad \text { and } \quad C^{-}=\{x \in C, \alpha(x)<0\} .
$$

Then $\mathcal{C}$ is the circuit set of an oriented matroid (called an affine oriented matroid) of rank $d+1$ on $V$. In particular, an affine oriented matroid $\mathcal{M}$ is acyclic which means that it has no circuit $C$ with $C^{-}=\emptyset$.

A nice geometric interpretation of the signed circuits of an affine oriented matroid is the following. Every circuit is a minimal signed set of points such that the convex hull of the positive elements intersect the convex hull of the negative elements. Thus, the signed circuits of an affine point configuration are exactly the minimal Radon partitions of the set of points.

Note that the circuit $(a, b, c, d, \bar{e}, \bar{f})$ of an affine oriented matroid $\mathcal{M}$ of rank 4 may induce any of the four relative positions of the corresponding points $a, \ldots, f$ as shown in Fig. 3. Their final position depends on the rest of the circuits of $\mathcal{M}$. 


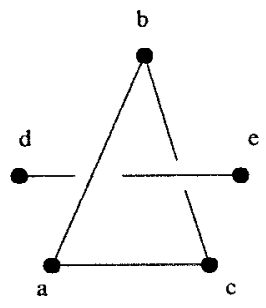

(i)

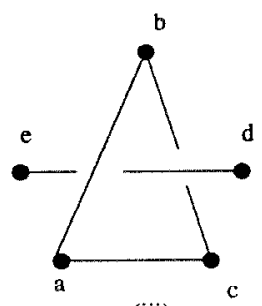

(iii)

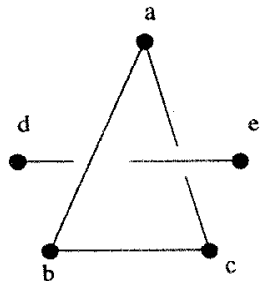

(ii)

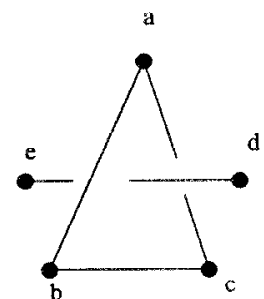

(iv)

Fig. 3. Possible point positions corresponding to the same circuit.

\section{Main Result}

We prove Theorem 1.2.

Proof of Theorem 1.2. Let $\mathcal{M}=\mathcal{M}\left[\mathcal{R}\left(\mathbf{K}_{7}\right)\right]$ be the affine oriented matroid arising from the set of points of a linear $\mathcal{R}\left(\mathbf{K}_{7}\right)$ where the points are labeled by $\{a, \ldots, g\}$. Suppose that $\mathcal{M}$ satisfies conditions $Q_{i}$ for some $1 \leq i \leq 3$ :

$$
\begin{aligned}
Q_{1}=\{ & (a, b, c, \bar{e}, \bar{f}) \in \mathcal{M},(a, f, b, \bar{c}, \bar{d}) \in \mathcal{M},(c, d, e, \bar{a}, \bar{b}) \in \mathcal{M}, \\
& (b, c, d, \bar{e}, \bar{f}) \in \mathcal{M},(b, c, d, \bar{a}, \bar{g}) \notin \mathcal{M},(b, c, d, \bar{f}, \bar{g}) \notin \mathcal{M}, \\
& (d, e, f, \bar{a}, \bar{g}) \notin \mathcal{M},(c, d, e, \bar{a}, \bar{g}) \notin \mathcal{M},(c, d, e, \bar{f}, \bar{g}) \notin \mathcal{M}\}, \\
Q_{2}= & (a, b, c, \bar{e}, \bar{f}) \in \mathcal{M},(c, d, e, \bar{a}, \bar{b}) \in \mathcal{M},(a, b, f, \bar{c}, \bar{d}) \notin \mathcal{M}, \\
& (a, b, f, \bar{d}, \bar{e}) \notin \mathcal{M},(a, f, g, \bar{c}, \bar{d}) \in \mathcal{M},(c, d, e, \bar{a}, \bar{g}) \notin \mathcal{M}, \\
& (a, b, f, \bar{g}, \bar{c}) \notin \mathcal{M}\}, \\
Q_{3}= & ((a, b, c, \bar{e}, \bar{f}) \in \mathcal{M},(c, d, e, \bar{a}, \bar{b}) \in \mathcal{M},(a, b, f, \bar{c}, \bar{d}) \notin \mathcal{M}, \\
& (a, b, f, \bar{d}, \bar{e}) \notin \mathcal{M},(a, f, g, \bar{c}, \bar{b}) \in \mathcal{M},(c, d, e, \bar{a}, \bar{g}) \notin \mathcal{M}\} .
\end{aligned}
$$

We claim that $\mathcal{R}\left(\mathbf{K}_{7}\right)$ contains a cycle isotopic to either $T$ or $T^{*}$. We consider three cases (according to the conditions $Q_{i}$ satisfied by $\mathcal{M}$ ). Each case is divided into four subcases (according to the relative position of elements $a, b, c, e, f$ induced by circuit $(a, b, c, \bar{e}, \bar{f}))$, see Fig. 3. In each subcase we show that the corresponding configuration 
of points induces a cycle isotopic to either $T$ or $T^{*}$. We do so by fixing the position of points $a, b, c, e, f$ and by adding first point $d$ and then point $g$ in such a way that the set of points satisfies the corresponding set of conditions $Q_{i}$. In fact, we only have to consider two subcases since Figs. 3(i) and (iv) are mirror images (similarly for Figs. 3(ii) and (iii)). Indeed, if we can add points $d$ and $g$ to the already fixed points $a, b, c, e, f$ (say, corresponding to Fig. 3(i)) in such a way that the configuration of points $a, b, c, d, e, f, g$ satisfies conditions $Q_{i}$ and induces $T$ (resp. $T^{*}$ ), then the configuration of points, arising from the mirror image of points $a, b, c, d, e, f, g$ (and hence $a, b, c, e, f$ correspond to Fig. 3(iv)), satisfies conditions $Q_{i}$ and induces $T^{*}$ (resp. $T$ ) as well.

Case I: $\mathcal{M}$ satisfies conditions $Q_{1}$. Subcases (a) and (b) are shown in Fig. 4.

Case II: $\mathcal{M}$ satisfies conditions $Q_{2}$. Subcases (a1), $(b, c, d, \bar{e}, \bar{f}) \in \mathcal{M}$, and (a2), $(b, c, d, \bar{e}, \bar{f}) \notin \mathcal{M}$, are shown in Fig. 5. Note that $(c, d, e, \bar{a}, \bar{f}) \in \mathcal{M}$ since $(c, d, e, \bar{a}$, $\bar{b}) \in \mathcal{M}$ and $(a, b, f, \bar{c}, \bar{d}) \notin \mathcal{M}$. Subcases (b1), $(a, c, d, \bar{e}, \bar{f}) \in \mathcal{M}$, and (b2), $(a, c, d, \bar{e}, \bar{f}) \notin \mathcal{M}$, are shown in Fig. 6 . Note that $(c, d, e, \bar{b}, \bar{f}) \in \mathcal{M}$ since $(c, d, e, \bar{a}$, $\bar{b}) \in \mathcal{M}$ and $(a, b, f, \bar{c}, \bar{d}) \notin \mathcal{M}$.

Case III: $\mathcal{M}$ satisfies conditions $Q_{3}$. Subcases (a1), $(b, c, d, \bar{e}, \bar{f}) \in \mathcal{M}$, and (a2), $(b, c, d, \bar{e}, \bar{f}) \notin \mathcal{M}$, are shown in Fig. 7 . Note that $(c, d, e, \bar{a}, \bar{f}) \in \mathcal{M}$ since $(c, d, e, \bar{a}$, $\bar{b}) \in \mathcal{M}$ and $(a, b, f, \bar{c}, \bar{d}) \notin \mathcal{M}$. Subcases (b1), $(a, c, d, \bar{e}, \bar{f}) \in \mathcal{M}$, and (b2), $(a, c, d, \bar{e}, \bar{f}) \notin \mathcal{M}$, are shown in Fig. 8. Note that $(c, d, e, \bar{b}, \bar{f}) \in \mathcal{M}$ since $(c, d, e, \bar{a}$, $\bar{b}) \in \mathcal{M}$ and $(a, b, f, \bar{c}, \bar{d}) \notin \mathcal{M}$.

Since any linear $\mathcal{R}\left(\mathbf{K}_{7}\right)$ give a uniform affine oriented matroid then it is sufficient to check that all uniform acyclic oriented matroid of rank 4 on seven elements always satisfy conditions $Q_{i}$ for some $1 \leq i \leq 3$ (possibly after a suitable permutation of elements). Indeed, this is true as was fully verified with the help of a computer program (see the Appendix).

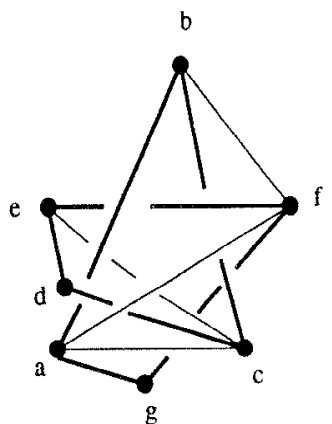

(a)

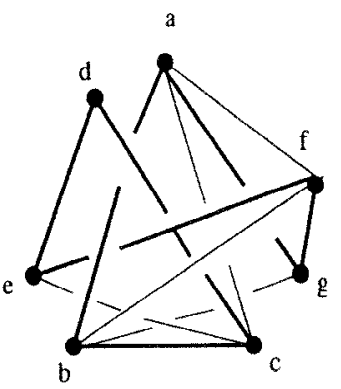

(b)

Fig. 4. 


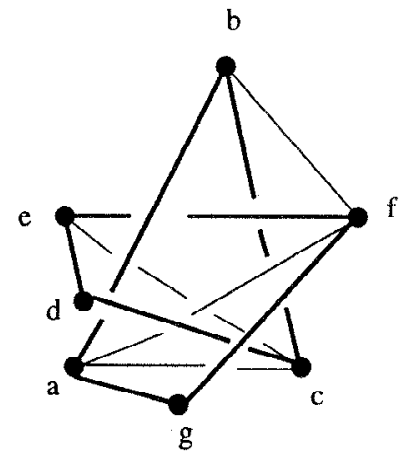

(a1)

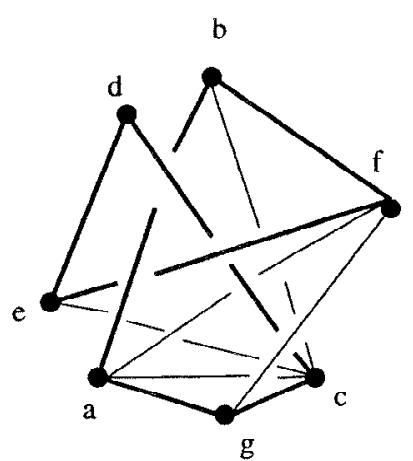

(a2)

Fig. 5.

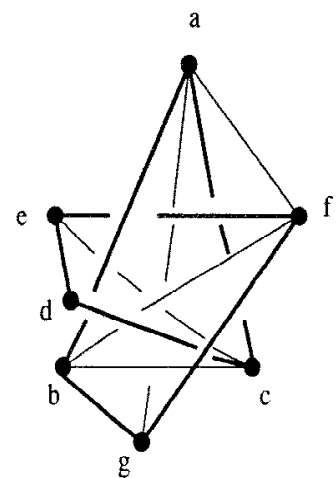

(b1)

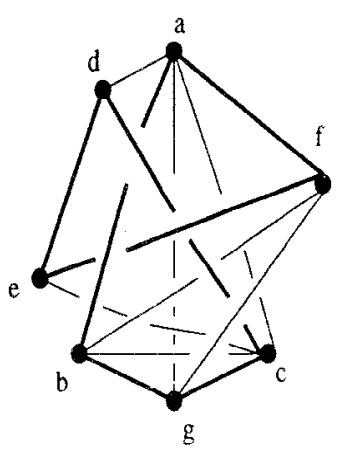

(b2)

Fig. 6.
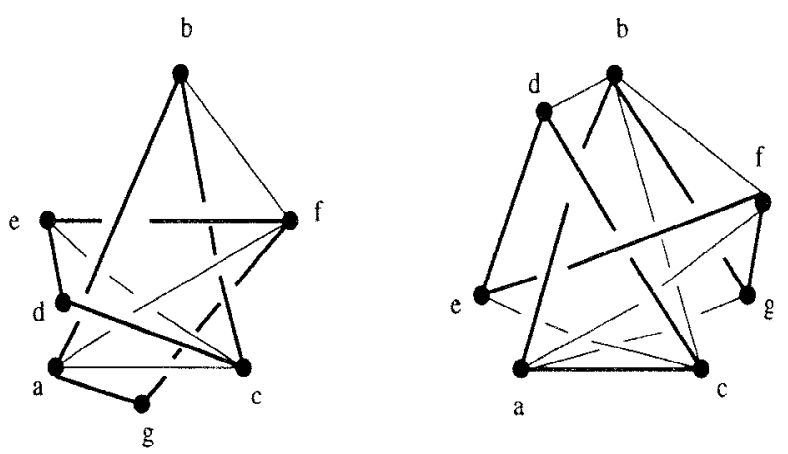

Fig. 7. 


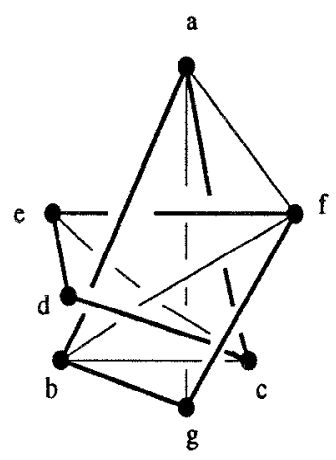

(b1)

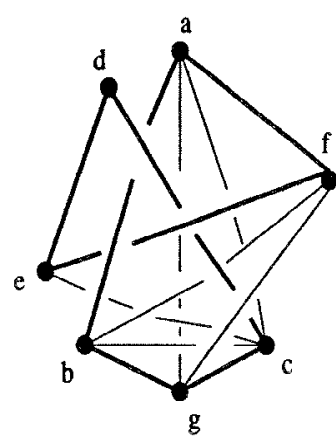

(b2)

Fig. 8.

A similar checking has also been done for all uniform acyclic oriented matroids of rank 4 on six elements. In this case we just consider the following set of conditions $Q_{4}$ :

$$
\begin{aligned}
& Q_{4}=\{(\bar{a}, b, c, \bar{d}, e) \in \mathcal{M},(a, \bar{b}, \bar{c}, d, f) \in \mathcal{M},(a, b, c, \bar{e}, \bar{f}) \in \mathcal{M}, \\
&(a, b, d, \bar{e}, \bar{f}) \in \mathcal{M},(\bar{a}, c, \bar{d}, e, f) \in \mathcal{M},(\bar{b}, \bar{c}, d, e, f) \in \mathcal{M}\} .
\end{aligned}
$$

Notice that the set of circuits in $Q_{4}$ (together with their opposites) gives the complete list of circuits of the oriented matroid $\mathcal{M}$. Moreover, any representation of either $T$ or $T^{*}$ with six points (the minimum number of points required) gives an oriented matroid with the same set of circuits as $\mathcal{M}$ (up to a permutation of elements), see Fig. 2.

It was found that there were oriented matroids not satisfying conditions $Q_{4}$, that is, there are linear spatial representations of $\mathbf{K}_{6}$ containing neither $T$ nor $T^{*}$ (see the Appendix). Then $m$ (Trefoil) $=7$.

Also, we have investigated if any uniform acyclic oriented matroid of rank 4 on seven elements always contains a subset of six elements satisfying conditions $Q_{4}$ (possibly after a suitable permutation). In other words, is it true that any linear $\mathcal{R}\left(\mathbf{K}_{7}\right)$ contains either $T$ or $T^{*}$ on six of the vertices? We found that the anwer is negative, that is, there exists linear $\mathcal{R}\left(\mathbf{K}_{7}\right)$ not containing either $T$ or $T^{*}$ using only six of the vertices.

\section{Acknowledgments}

I would like to thank Michel Las Vergnas for welcoming me to his laboratory and for several discussions. I also thank the anonymous referees for helpful suggestions

\section{Appendix}

Here, we summarize the perfomance of the checking conditions program. 
Table 1. The chirotopes are ordered lexicographically.

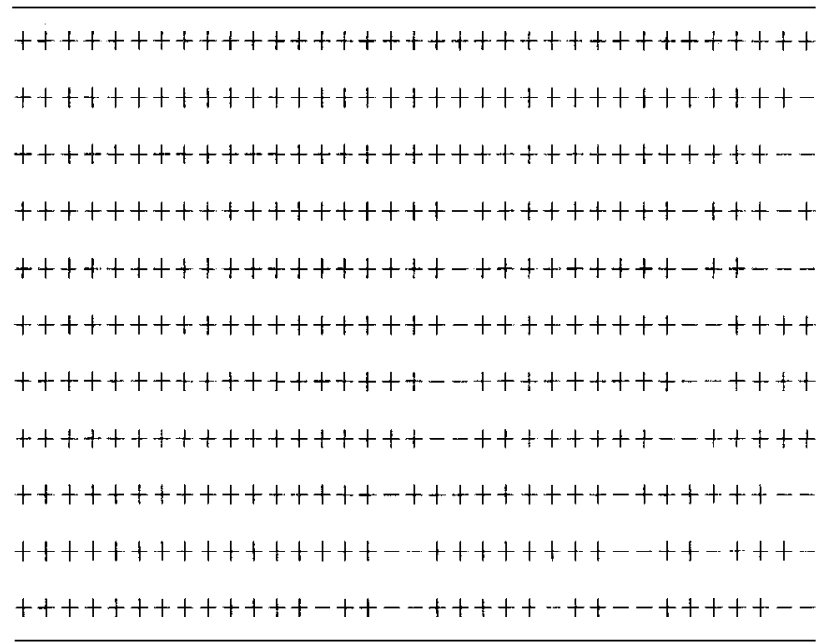

\section{Checking Conditions Program}

(1) Read a representative chirotope from each of the eleven different orientation classes arising from all the uniform oriented matroids of rank 4 on seven elements, see Table 1. Construct the corresponding set of circuits.

(2) Given the circuits, generate all the $2^{6}$ different oriented matroids in the class. This can be done by reorienting the elements corresponding to all the subsets of $\{1, \ldots, 7\}$ of size zero, one, two, and three.

(3) For each reorientation, verify that it gives an acyclic oriented matroid (ignore it otherwise as it is not an affine oriented matroid). If the reorientation is acyclic, then check if there is a suitable permutation of elements such that it satisfies one of the conditions $Q_{i}, 1 \leq i \leq 3$. Finally, output any acyclic oriented matroid not satisfying any of conditions $Q_{i}$.

The program does not output any oriented matroids. Therefore all acyclic oriented matroid verify at least one of conditions $Q_{i}$. Here, we remark that the three sets of conditions $Q_{i}$ are needed, that is, there are uniform oriented matroids of rank 4 on seven elements that satisfy only one of conditions $Q_{i}$.

We have run a similar program for uniform oriented matroids of rank 4 on six elements. In this case we just consider conditions $Q_{4}$ for the only orientation class given by $\chi(\lambda)=+$ where $\lambda$ is any of the $\left(\begin{array}{l}6 \\ 4\end{array}\right)$ four-element subset of $\{1, \ldots, 6\}$. Here, we found that among the $2^{5}$ different uniform oriented matroids (obtained by reorientations) only two (those obtained by reorienting $(3,4)$ and $(1,4,5)$ ) satisfied condition $Q_{4}$.

\section{References}

1. B. Björner, M. Las Vergnas, B. Sturmfels, N. White, and G. Ziegler, Oriented Matroids, Encyclopedia of Mathematics and its Applications, Addison-Wesley, Reading, MA, 1993. 
2. J.A. Bondy and U.S.R. Murty, Graph Theory and its Applications, MacMillan, New York, 1970.

3. G. Burden and A. Zieschang, Knots, Studies in Mathematics, Vol. 15, de Gruyter, New York, 1985.

4. J.H. Conway and C.McA. Gordon, Knots on spatial graphs, J. Graph Theory, 7 (1983), 445-453.

5. M. Dehn, Die beiden Kleeblattschlingen, Math. Ann., 69 (1914), 402-413.

6. J. Folkman and J. Lawrence, Oriented matroids, J. Combin. Theory Ser. B, 25 (1978), 199-236.

7. J. Lawrence, Oriented matroids and multiply ordered sets, Linear Algebra Appl., 48 (1982), 1-12.

8. M.S. Miyauchi, Topological Ramsey theorem for complete bipartite graphs, J. Combin. Theory Ser. B, 62 (1994), 164-179.

9. S. Negami, Ramsey theorems for knots, links and spatial graphs, Trans. Amer. Math. Soc., (1991), 527-541.

10. K. Reideimeister, Homotopieringe und Linsenräume, Sem. Hamburg, 11 (1936), 102-109.

11. N. Robertson, P. Seymour, and R. Thomas, Kuratowski chains, J. Combin. Theory Ser. B, 64 (1995), 127-154.

12. N. Robertson, P. Seymour, and R. Thomas, Petersen family minors, J. Combin. Theory Ser. B, 64 (1995), 155-184.

13. N. Robertson, P. Seymour, and R. Thomas, Sacks' linkless embedding conjecture, J. Combin. Theory Ser. B, 64 (1995), 185-227.

14. H. Sacks, On spatial representation of finite graphs, in Finite and Infinite Sets, Colloquial Mathematica Societati Janos Bolyai, Vol. 37, North-Holland, Amsterdam, 1981.

Received July 8, 1997, and in revised form January 16, 1998. 